\title{
Single-Objective Optimization Methodology for the Design of RF Integrated Inductors
}

\author{
Fábio Passos ${ }^{1}$, Maria Helena Fino ${ }^{1}$, and Elisenda Roca ${ }^{2}$ \\ ${ }^{1}$ Faculdade de Ciências e Tecnologia, Universidade Nova de Lisboa \\ 2829-516 Caparica, Portugal \\ f.passos@campus.fct.unl.pt, hfino@fct.unl.pt \\ ${ }^{2}$ Instituto de Microelctrónica de Sevilla, CSIC and Universidade de Sevilla, \\ Seville, Spain \\ eli@imse-cnm.csic.es
}

\begin{abstract}
Designing integrated inductors for RF applications is quite a challenging task due to the necessity of minimizing the parasitic effects arising from using today's technologies. The multiplicity of non-ideal effects to be minimized makes imperious the use of optimization-based design methodologies. In this paper a model-based optimization methodology is considered as a way of offering the designer the possibility to obtain inductors with maximum quality factor. The inductor model accounts for square, hexagonal or octagonal topologies. Furthermore tapered inductors are also accounted for in the proposed model. The use of the inductor model reduces the optimization time significantly. The validity of the results obtained is checked against electromagnetic (EM) simulations. As an application example, the particular case for the design of inductors for $2.4 \mathrm{GHz}$ is illustrated.
\end{abstract}

Keywords: Integrated Inductors, RF, Design, Optimization etc.

\section{Introduction}

Integrated Inductors are a very important element on modern radio frequency (RF) design [1]. During the past few years, the design of integrated inductors has attracted much interest in both IC design and electronic design automation communities. In order to design an integrated inductor three main parameters must be considered by the designer [2]. One of the most critical parameter, if not the most critical one, is inductance value. Afterwards, quality factor (Q) and self-resonance frequency (SRF) [3] are to be taken into consideration.

Given an inductor design, different approaches may be followed in order to evaluate these parameters. The most common and widely used are: electromagnetic (EM) simulations, surrogate models and lumped element models.

EM simulations are time consuming, i.e. a simulation of a single inductor in a visual environment with ADS Momentum [4] can last up to eight hours. However this is the most accurate simulation possible when designing inductors. Surrogate models i.e. [5] are mathematically extensive, computing exhausting and they are not 
physically understandable, and they introduce errors due to the mathematical expressions. On the other hand, lumped element models are based on physical expressions, which allow a much easier interpretation of the models [6]. Furthermore, as they rely on geometrical and technological parameters, design migration to new technologies is a straightforward process. In the end, these lumped element models are the option that allows the best trade-off between accuracy and simulation complexity. In order to obtain an inductor with desired impedance or the maximum quality factor possible, optimization based simulations must be used. It is possible to understand that if EM simulations and surrogate models are time prohibitive for only one inductor, to integrate these evaluation tools into optimization loops, is not a practicable solution. These leave designers with only one solution that is the usage of lumped element models. In this work the common $\pi$-model is used to model integrated inductors. After presenting the main contribution of the work to the implementation of collective Awareness systems, the inductor model is briefly described. Then, the integration of the inductor model into an optimization tools is presented. For the optimization a selection based differential evolution algorithm (SDBE) is used. Validity of the proposed methodology is performed by comparing the results of the optimization process for the design of an integrated inductor for 2.4 $\mathrm{GHz}$ against EM simulations. Finally conclusions are driven.

\section{Relationship to Collective Awareness Systems}

Collective Awareness systems link objects, people and knowledge in order to foster new forms of social and business innovation. The implementation of such systems relies on networks supporting the interconnections of a large number of heterogeneous cooperating devices. The development of systems has been made possible due to the rapid evolution of electronic technologies, which enable the implementation of ever more complex functions, in smaller and more rapid and less consuming circuits. To cope with the necessity of minimizing the power consumption of such systems, new design methodologies must be adopted so that the ever more stringent specifications may be attained. In the particular case of communications services, e.g. wireless communications devices, integrated inductors are becoming widely used in either Voltage Controlled Oscillators, LNAs and other RF blocks.

The mains contribution of this paper is the definition of a single-objective modelbased optimization methodology for the design of integrated inductors. The main added value relies on the possibility for designing square, hexagonal or octagonal, tapered/no tapered inductors.

\section{$3 \quad$ Integrated Inductor Model}

The simple p-model is the most widely used for characterizing integrated inductor. Its applicability, however is limited to frequencies in the order of $1 \mathrm{GHz}$. F The model used in this work is based on the segment model approach [7]. The inductor is divided into segments and then each segment is characterized with the $\pi$ lumped-element 
circuit shown in Fig. 1. This figure illustrates a typical square integrated inductor, where $n$ is the number of turns, $w$, the width of the metal turn, $s$, the spacing between metal turns, $D_{\text {out }}$, the outer diameter and, finally, $D_{i n}$, the inner diameter.

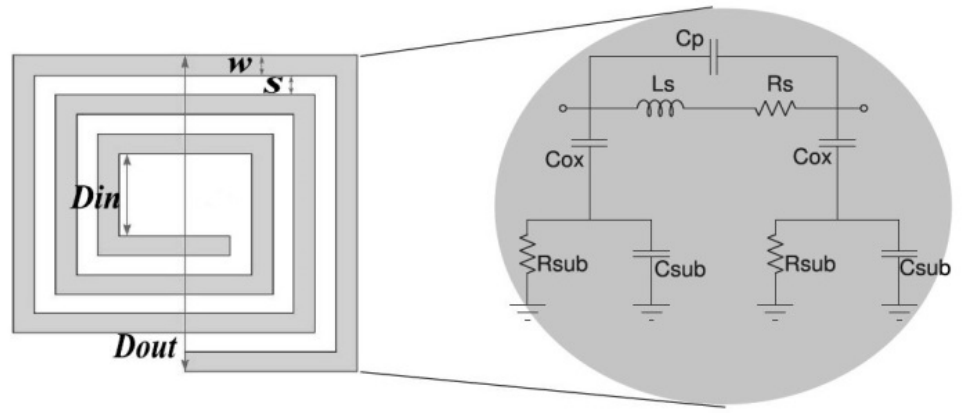

Fig. 1. Square inductor, its physical parameters and the $\pi$ model

The series branch of this model consists of $L_{S}, R_{S}$ and $C_{S}$. The series resistance, $R_{S}$ arises from metal resistivity of the inductor and is closely related to the quality factor, being a key issue for inductor modeling. The series feed-forward capacitance, $C_{p}$, is usually considered as the overlap capacitance between the spirals and the underpass metal lines, also called $C_{O}$ [8]. However, as the minimum feature size of CMOS process continues to shrink, the spacing between metal spirals, $s$, can be reduced to a value similar to the distance to the underpass. Therefore the coupling capacitance between metal lines, $C_{S}$, can increase its significance in the total capacitance of the device, and therefore in the self-resonance frequency (SRF). $C_{p}$ is then given by the sum of $C_{S}$ and $C_{O}$. The capacitance $C_{O X}$ represents the oxide capacitance between the spiral and the substrate. The silicon substrate is modeled with $C_{s u b}$ and $R_{S u b}$ [9]. A detailed explanation on how to evaluate $L_{S}$ may is presented in [10]. For the remaining elements analytical expressions may be obtained in [11].

For the sake of simplicity some physical aspects were not taken into account in the analytical characterization of the model elements. For example, the model does not take into account Eddy currents [8], thus for small inner diameters, the model is less accurate. Also, with a wider metal, the parasitic capacitances and fringing capacitances tend to increase and again, induce higher errors. Since this model is to be integrated into an optimization based design tool, the validity of the model along the overall design space was performed through a statistical analysis [11].

\section{$4 \quad$ Optimization of Integrated Inductors}

This section discusses the optimization of integrated inductors in a $0.35 \mu \mathrm{m}$ CMOS technology for $2.4 \mathrm{GHz}$. In this work an octagonal layout is simulated because it uses less metal to draw turns than square or hexagonal layouts, and can therefore achieve 
higher quality factors. This section presents a single-objective optimization using the selection based differential evolution (SBDE) algorithm is presented. The results of the optimization processes are validated against EM simulations.

For inductor optimization, common design variables are the geometric parameters (i.e. number of turns, the inner diameter, the turn width and the spacing between turns) and the objectives are the inductor performances at a certain frequency (i.e. equivalent inductance, quality factor, self-resonance frequency and area). For the specific case of inductor optimization the SRF may not be explicitly defined as an objective. The guaranty that the SRF is well above the operating frequency may be obtained through the definition of some specific constraints such as the imposition that the inductor has to be in the plan bandwidth zone, i.e., the frequency band where the inductance value remains within $10 \%$ from the DC value [15].

In the case of the single objective, if several objectives envisaged into one optimization process, it is possible to build an objective function reflecting the weighted sum of the several objective functions.

The general constraints imposed in the optimization processes are:

- The inductance value is limited to only 5\% error from the inductance desired at $2.4 \mathrm{GHz}$,

- The inductance curve has to be in the plain bandwidth zone,

- The quality factor has to be before the peak value, which means that the quality factor has to be in the positive slope region of the quality factor.

The single-objective optimization was made with the objective of finding an inductor with a given inductance value, while maximizing the quality factor and minimizing the area. This was possible through the weighted objective function shown in (1)

$$
\text { Objective }=\operatorname{Min}(-(0.7 \cdot Q)+0.3 \cdot \text { Area })
$$

Several simulations were made in order to obtain inductors with a desired inductance for $1 \mathrm{nH}$ to $5 \mathrm{nH}$. All optimizations were done with 300 individuals and 300 iterations and the simulation time was about 300 seconds per simulation. The inductors obtained were then simulated with ADS Momentum, in order to check the validity of the results. The comparison values and curves are shown for each of the inductors obtained. The physical parameters of the inductors are given in Table 2, as well as the values for inductance and quality factor at $2.4 \mathrm{GHz}$. All the physical parameters are given in $\mu \mathrm{m}$, and the inductance in $\mathrm{nH}$. The relative error $(\varepsilon)$ is given in \%. The physical parameters also had to be limited so that the model is accurate over the design space considered. So, the geometric parameters were limited according to the values shown in Table 1. Layout and fabrication processes impose limits on the precision of the physical dimensions, and so not all values in a range can be used, only some discrete values. These values are defined by the grid of the optimization process and are related to each technology. 
Table 1. Restrictions on the inductor physical parameters

\begin{tabular}{c|ll}
\hline Parameter & Minimum & Maximum \\
\hline$n$ & 2 & 7 \\
$\boldsymbol{D}_{\text {in }}(\mu \mathrm{m})$ & 100 & 250 \\
$w(\mu \mathrm{m})$ & 5 & 10 \\
\hline
\end{tabular}

Table 2. Comparison between inductance and quality factor for optimized inductors

\begin{tabular}{c|llllllllll}
\hline $\boldsymbol{L}_{\boldsymbol{D}}$ & $n$ & $w$ & $\boldsymbol{D}_{\boldsymbol{i n}}$ & $s$ & $\boldsymbol{L}_{\boldsymbol{M o d e l}}$ & $\boldsymbol{L}_{\boldsymbol{E M}}$ & $\varepsilon$ & $\boldsymbol{Q}_{\text {Model }}$ & $\boldsymbol{Q}_{\boldsymbol{E M M}}$ & $\varepsilon$ \\
\hline $1 \mathrm{nH}$ & 2 & 10 & 123 & 2.5 & 1.04 & 1.01 & $\mathbf{2 . 9 7}$ & 8.80 & 9.07 & $\mathbf{2 . 9 8}$ \\
$2 \mathrm{nH}$ & 3 & 10 & 106 & 2.5 & 2.10 & 1.87 & $\mathbf{1 2 . 3}$ & 11.17 & 9.85 & $\mathbf{1 3 . 4}$ \\
$3 \mathrm{nH}$ & 3 & 10 & 144 & 2.5 & 2.80 & 2.66 & $\mathbf{5 . 2 6}$ & 11.32 & 10.35 & $\mathbf{9 . 3 7}$ \\
$4 \mathrm{nH}$ & 3 & 8.03 & 185 & 2.5 & 3.77 & 3.71 & $\mathbf{1 . 6 2}$ & 10.24 & 1.10 & $\mathbf{1 . 3 9}$ \\
$5 \mathrm{nH}$ & 4 & 5.63 & 143 & 2.5 & 5.03 & 4.90 & $\mathbf{2 . 6 5}$ & 9.21 & 9.55 & $\mathbf{3 . 5 6}$ \\
\hline
\end{tabular}

It is possible to conclude that the model can be used in RF frequency range in the design space where the optimization process was developed. The errors observed in the optimization process are usually quite acceptable. It is also possible to observe that the inductor that has the highest error has the Din and the $w$ close to its boundaries, which increases the error values.

\section{Conclusions and Future Work}

In this work an efficient lumped-element model to characterize integrated inductors is presented. The model uses analytical expressions to evaluate the lumped elements values. The model is able to predict the inductance and the quality factor values with relative accuracy. The model was also integrated into an optimization process and the results validated against EM simulation. It has been proved that the model is suitable for RF circuit design and its best feature is the ability to save time in the design process. As future work, the model can be fitted to smaller frequency ranges in order to obtain higher accuracy. The complexity of the model can also be increased, adding elements to the equivalent circuit.

\section{References}

1. Niknekad, A.M.: Electromagnetics for High-Speed Analog and Digital Communication Circuits (2007)

2. Zhan, Y., Sapatnekar, S.S.: Optimization of integrated spiral inductors using sequential quadratic programming. In: Proceedings of the Design, Automation and Test in Europe Conference and Exhibition, February 16-20, vol. 1, pp. 622-627 (2004)

3. Mohan, S.S.: The Design, Modeling and Optimization of On-chip Inductor and Transformer Circuits. Stanford University, Dept. of Electrical Engineering (1999)

4. ADS Momentum. Agilent technologies. EEsof division, Santa Rosa, CA (2006) 
5. Nieuwoudt, A., Massoud, Y.: Variability-Aware Multilevel Integrated Spiral Inductor Synthesis. IEEE Transactions on Computer-Aided Design of Integrated Circuits and Systems 25(12), 2613-2625 (2006)

6. Passos, F., Helena Fino, M., Roca, E.: A Wideband Lumped-Element Model for Arbitrarily Shaped Integrated Inductors. In: IEEE ECCTD Conference Proceedings (2013)

7. Koutsoyannopoulos, Y., Papananos, Y., Alemanni, C., Bantas, S.: A generic CAD model for arbitrarily shaped and multi-layer integrated inductors on silicon substrates. In: Proceedings of the 23rd European Solid-State Circuits Conference, ESSCIRC 1997, pp. 320-323 (September 1997)

8. Yue, C., Wong, S.: Physical modeling of spiral inductors on silicon. IEEE J. Transactions on Electron Devices 47, 560-568 (2000)

9. Lee, T.H.: The Design of CMOS Radio-Frequency Integrated Circuits. Cambridge University Press (2004)

10. Passos, F., Helena Fino, M., Roca, E.: Analythical Characterization of Variable Width Integrated Spiral Inductors. In: IEEE MIXDES Conference Proceedings (2013)

11. Passos, F., Helena Fino, M., Roca, E.: Lumped Element Model for Arbitrarily Shaped Integrated Inductors - A Statistical Analysis. In: IEEE COMCAS Conference Proceedings (2013)

12. Greenhouse, H.: Design of planar rectangular microelectronic inductors. IEEE J. Trans. PHP 10(2), 101-109 (1974)

13. Grover, F.W.: Inductance calculations: Working formulas and tables. Courier Dover Publications (1929)

14. Yue, C., Wong, S.: On-chip spiral inductors with patterned ground shields for Si-based RF ICs. IEEE Journal of Solid-State Circuits 33(5), 743-752 (1998)

15. Sieiro, J., Lopez-Villegas, J.M., Osorio, J.A., Carrasco, T., Vidal, M.N., Ahyoune, S.: Synthesis of compact planar inductors in LTCC technology. In: 2012 International Conference on Synthesis, Modeling, Analysis and Simulation Methods and Applications to Circuit Design (SMACD), pp. 45-48 (2012) 